\title{
«lch bin die Stimme der Praxis»
}

\section{Daniel Lüthi}

Freier Journalist und Fotograf, Medientrainer, Bern

Das Bild ist symbolisch: Selbstbewusst und zielstrebig schreitet eine Pflegefachfrau - Schülerin vielleicht noch - über den Rasen vor der Schule. Die Dozentin blickt, bewundernd fast, zu ihr empor. «Ja», sagt Pia Coppex, «wir dürfen stolz sein auf unseren Beruf.» Die Skulptur von Denis Perret-Gentil gefällt ihr vor allem wegen ihrer Aussage.

\section{Eine Pflegende als Präsidentin}

An der Fachhochschule «La Source» in Lausanne ist Pia Coppex nicht nur Dozentin, sondern seit knapp einem Jahr auch für den DAS-Studiengang «Gesundheit im
Alter» verantwortlich. National hat sie jedoch wegen eines anderen Amtes Aufsehen erregt: Sie ist die erste Pflegefachfrau, die Präsidentin der Schweizerischen Gesellschaft für Biomedizinische Ethik SGBE geworden ist. Im April wurde sie an die Spitze eines Gremiums gewählt, das über 20 Mitglieder zählt und zahlreiche Titel vereint: Prof. Dr. med., Prof. Dr. theol., Dr. phil., dipl. psych., Dr. iur. und so weiter.

Pia Coppex hat keinen Doktortitel, aber eine solide Ausbildung und viel Erfahrung. Sie betont, sie sei in ihrer professionellen Laufbahn immer nahe der Basis geblieben, bei den Leuten "du terrain» und dem, was diese beschäftigt. «Ich bin die Stimme der Praxis», sagt

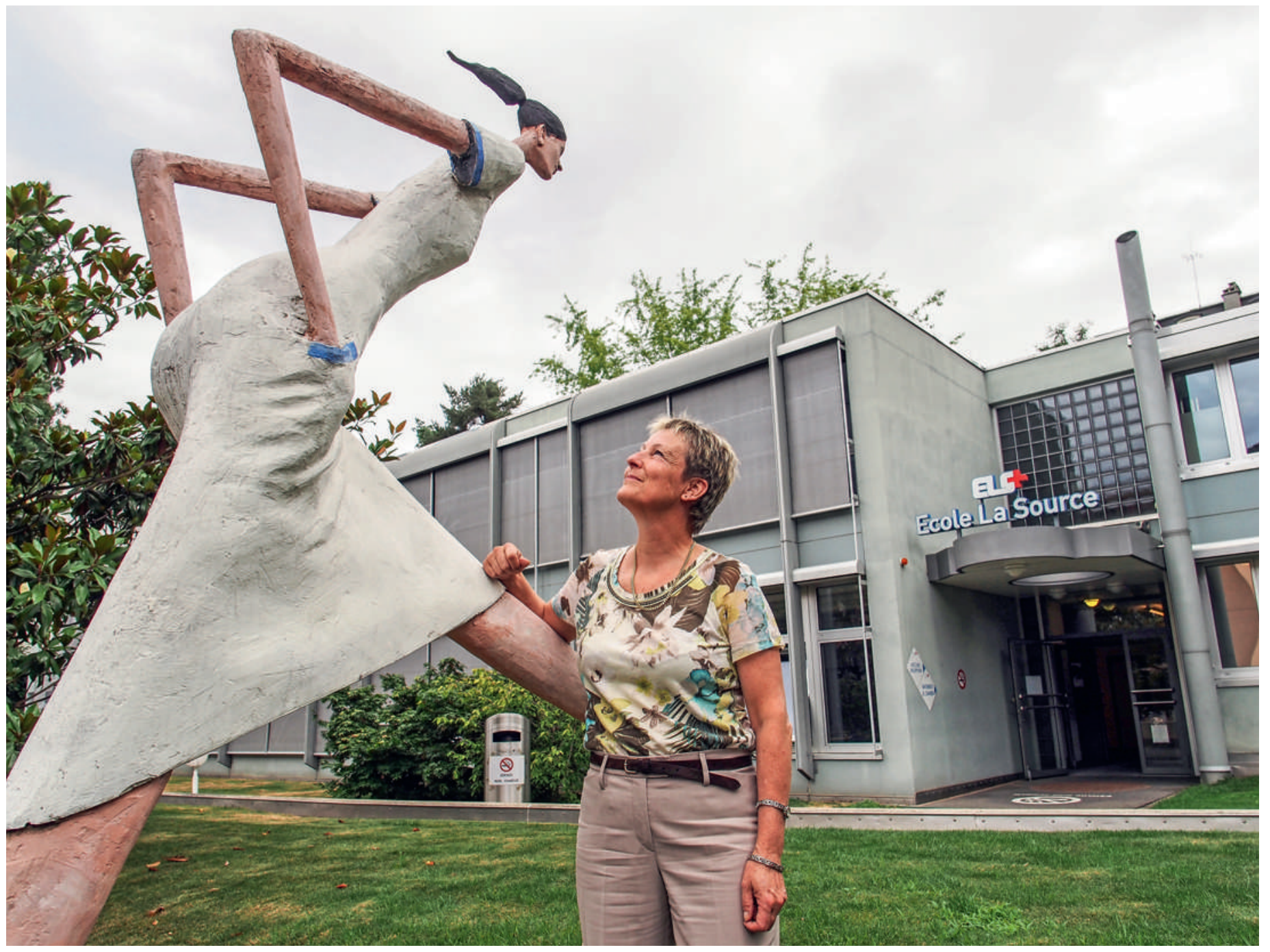


sie, «und ich fühlte mich geehrt, dass man bei der Wahl an mich gedacht hat.» Warum wollte sie dieses Amt und damit eine zusätzliche Verantwortung übernehmen? «Das Gesundheitswesen ist komplex geworden da ist es mir ein Anliegen, die verschiedenen Akteure zusammenzubringen. Und: In einer Zeit, in der wirtschaftliche Argumente immer mehr Gewicht haben, ist es wichtig, andere Werte einzubringen.» Darum geht es ihr, wenn sie von Ethik spricht, um Werte wie Verantwortung, Menschenrechte, Würde und Respekt. Werte, die für sie auch am Patientenbett immer im Vordergrund standen.

\section{Was macht Sinn?}

Ein Beispiel: Pia Coppex erzählt von einer Frau, die zuhause lebte, aber zunehmend dement war. Immer weniger war sie fähig, zu sich selber zu schauen, kaum mehr kochte sie. Sie stürzte und verletzte sich, sie verbrannte sich mit heissem Kaffee. «Ihre Nichte schlug Alarm, aber die Frau wollte zuhause bleiben.» Eine Entscheidung drängte sich auf und damit eine Absprache aller Beteiligten: Hausarzt, Pflegende, Nichte - und betroffene Frau. «In einem solchen Fall darf man sich nicht auf formalistische Aspekte beschränken, vor allem aber darf man eine Person und ihre Angehörigen nicht fallen lassen.» Was macht in diesem Moment für diese Person Sinn, was trägt zu ihrem Wohlergehen wirklich bei? Das sei die Frage.

\section{«Es geht darum, verschiedene Blickwinkel zusammenzubringen.»}

Dazu ein zweites Beispiel: Eine Frau brauchte Physiotherapie, um wieder richtig gehen zu lernen, um ihre Muskeln zu stärken, um zu üben. Eine Kollegin von Pia Coppex setzte die Dame in einen Rollstuhl, um sie in die Physiotherapie zu fahren. "Das ist doch völliger Nonsense», entgeistert sich Coppex, "da hat sich jemand einfach keine Fragen gestellt. Es reicht eben nicht, bloss zu sagen: 'Ich mache das für dein Wohl'. Manchmal ist das Richtige ganz alltäglich und naheliegend.»

\section{Das Team im Zentrum}

Natürlich sei es nicht immer einfach, den idealen Weg zu finden, im Gesundheitswesen würden die Akteure immer wieder mit menschlich schwierigen Situationen konfrontiert, wo es kein Patentrezept gebe. Deshalb sei eben die Teamarbeit so zentral: «Ein Team, das gelernt hat zusammenzuarbeiten, profitiert schon beim nächsten Entscheid davon.» Sie selber habe hier-

\section{Pia Coppex-Gasche}

Pia Coppex-Gasche wurde 1961 in Solothurn geboren. Mit sechs Jahren zog sie mit ihrer Familie nach Monthey im Unterwallis, wo sie die Schulen besuchte und ihre Ausbildung als Psychiatrie-Krankenschwester - so hiess das damals noch - absolvierte. 1983 wurde sie diplomiert, dann arbeitete

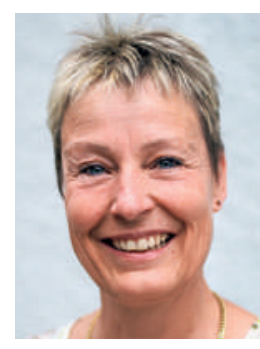
sie drei Jahre lang in der Notfallabteilung des Spitals in Monthey. Es folgten verschiedene Weiterbildungen, insbesondere in den Bereichen Gerontologie, Psychologie des Alters, Gesundheitsrecht, Pädagogik und Ethik. 2011 bis 2014 arbeitete sie als Projektleiterin bei der Gesundheitsdirektoren-Konferenz GDK in Bern. Seit September 2014 ist sie an der Fachhochschule "La Source" in Lausanne tätig - als Dozentin und Verantwortliche für den Studiengang "Gesundheit im Alter». Im April 2015 wurde sie - als erste Pflegefachfrau - zur Präsidentin der Schweizerischen Gesellschaft für Biomedizinische Ethik gewählt. Pia Coppex ist verheiratet und Mutter von zwei erwachsenen Töchtern. Sie lebt mit ihrem Mann in Choëx oberhalb von Monthey.

archische Hürden zwischen Pflegenden und Ärzteschaft oder Standesdünkel bei Medizinern nicht erlebt. «In der Psychiatrie war die interdisziplinäre Zusammenarbeit schon während meiner Ausbildung selbstverständlich.» Deshalb schaue sie auch heute nicht ehrfürchtig zu den Medizinern empor. "Ärzte sind meine Kollegen.» Und alle anderen, denen das Wohl der Patienten am Herzen liegt. «Ich hatte immer wieder schöne Begegnungen, und viele Berufskolleginnen und -kollegen sind heute meine Freunde», erzählt Pia Coppex.

In nächster Zeit will die neue SGBE-Präsidentin jedes Vorstandsmitglied einzeln treffen und kennen lernen. Ganz nach dem Motto dieser Gesellschaft. Sie sei 1989 gegründet worden, schreibt sie, um «die Verständigung über die Grenzen der einzelnen Fachdisziplinen hinaus zu suchen». Offensichtlich keine Selbstverständlichkeit.

«Es geht darum, verschiedene Blickwinkel zusammenzubringen. Es geht um den Dialog - mit dem Ziel, sich $\mathrm{zu}$ verstehen und $\mathrm{zu}$ einigen, also einen Konsens zu finden.» Ein Kompromiss sei nicht immer möglich oder nötig, erklärt Pia Coppex und illustriert das gesagte Abstrakte auf Wunsch wiederum mit einem konkreten Beispiel. Es ging um die Frage, ob die Fachleute bei einer jungen Frau eine Abtreibung befürworten sollten oder nicht. «Meine ursprüngliche Meinung war das Gegenteil des späteren Entscheids. Aber dieser war für mich dann ok, weil wir ihn in der Equipe erarbeitet und eine solidarische Haltung entwickelt hatten.» 


\section{Der graue Tsunami ...}

Pia Coppex ist spezialisiert auf Themen des Alters, darunter Demenz und Palliativcare. Themen, notabene, die Gesundheitsexperten permanent die eigenen Grenzen und die eigene Vergänglichkeit vor Augen führen. Warum entschied sie sich als junge Frau gerade dafür? «Unsere Demographie ist, wie sie ist, der 'graue Tsunami' ist eine Tatsache und Herausforderung», antwortet sie nach einiger Überlegung. «Wir müssen unsere Ressourcen künftig sehr effizient einsetzen, wenn wir mit unserem Gesundheitssystem in den nächsten Jahren den Bedürfnissen der Bevölkerung noch gerecht werden wollen.»

\section{«Schön wäre es, für die Weltgesundheits- organisation WHO zu arbeiten.»}

Dann blickt sie, wie fast immer in einem solchen Moment, vielsagend in die Weite, sucht den Gedanken und entscheidet spontan, ob sie ihn auf Schweizerdeutsch oder Französisch formulieren will. Jetzt atmet sie tief ein und blickt das Gegenüber lächelnd an: «Alte Leute haben mir immer so viel gebracht", schwärmt sie, «Demente auch. Sie haben vielleicht keine Worte mehr - aber diese Blicke! Und mit unseren Blicken und der Art, wie wir sie ansprechen, können wir erreichen, dass diese Personen Personen bleiben, Menschen mit einer Geschichte, einem Schicksal und einem Anspruch auf Autonomie - dass sie nicht zu Objekten werden.» Wieder schwebt der Begriff der menschlichen Würde im Raum und damit der oft wenig konkrete und schwer fassbare Themenbereich der Ethik.

\section{... und die Sache mit der Zeit}

Zeit haben, sich Zeit nehmen, um zuzuhören - das würde dazugehören. Mehr noch: Das wäre zentral. Bloss: Alle, die im Gesundheitswesen arbeiten, klagen über einen Mangel an Zeit. Pia Coppex sagt: «Sobald die Performance und damit auch die Administration im Zentrum stehen, müssen Pflegende sowie Ärztinnen und Ärzte oft entgegen ihren eigenen Werten und Überzeugungen arbeiten - mit dem Risiko, sich selber zu verlieren.» Aber sie sagt auch: «Die Frage der Zeit ist relativ. Zeit ist nicht eine Frage der Quantität, sondern der Qualität. Der Präsenz und der Verfügbarkeit. Manchmal reicht ein Blick, genügen ein paar wenige, aber aufrichtige Worte.» Entscheidend sei, dass eine Pflegende oder eine Ärztin sich selber nicht ausblende und authentisch bleibe.

Sich zu wehren sei schon richtig, sagt Coppex, eine Revolutionärin ist sie deswegen aber keineswegs. Sie bleibt freundlich und fröhlich, auch wenn sie bestimmt ist. Und sie sagt: «Im Zentrum steht der Patient. Um ihn sollen sich alle Mitglieder einer interprofessionellen Equipe bestmöglich kümmern. Dafür braucht es den Dialog.»

\section{Das Wallis und die Welt}

Im kleinen Büro, das sie mit jemandem teilt, hängt ein kleines Werbeplakat für das Wallis an der Wand. Mehrmals erwähnt sie es, das Wallis ist für sie der Herzensort, dort ist sie zu Hause. Sie pendelte nach Bern, und sie pendelt nach Lausanne. Ihre Familie und Freunde, der Garten und die Berge seien ihr wichtig, sagt sie, "und die Tatsache, dass man es im Wallis wagt, sich gegenseitig zu sagen, was Sache ist». Ihre Verankerung ist stark, beruflich und geographisch.

In der Freizeit fügt sie farbige Glasstücke mit Blei zu Bildern zusammen. Die Vielfarbigkeit ist symbolisch für diese Frau, auch in ihrem beruflichen Leben. Immer wieder bringt sie Leute aus verschiedenen Berufen zusammen, zur Zeit ist sie gerade daran, in Lausanne einen grossen interkantonalen Kongress auf die Beine zu stellen, zu ihrem Kernthema "Älter werden in der Schweiz». Pia Coppex ist eine Netzwerkerin, die von ihren Basen aus viel unterwegs ist, auch gedanklich. Sie ist also nicht eine Frau, die still steht, sondern immer weiter will, stolz voranschreitet, wie die Skulptur vor der Schule «La Source» in Lausanne.

Welches ist ihr nächstes Ziel? Coppex lacht: «Ich habe in verschiedenen Institutionen gearbeitet, auf kantonaler und nationaler Ebene. Schön wäre jetzt der Schritt in die internationale Gesundheitspolitik. Wenn ich ein wenig träumen darf: Schön wäre es, für die Weltgesundheitsorganisation WHO zu arbeiten.» Den Bezug zur Basis, zur Praxis würde sie sicher auch dort nicht verlieren wollen.

\section{Die nächste «Begegnung mit ...»}

Am Ende jeden Monats stellt die Schweizerische Ärztezeitung eine Persönlichkeit vor, die sich im Gesundheitswesen engagiert. Im September schildert Daniel Lüthi seine Begegnung mit Daniel Hell, emeritierter Psychiatrie-Professor. 